\title{
In situ Scanning/Transmission Electron Microscopy and Spectroscopy of Local Ionic Transport in Battery Electrodes
}

Feng Wang

Sustainable Energy Technologies Department, Brookhaven National Laboratory, Upton, NY 11973

* Corresponding author:fwang@bnl.gov

The design and development of safe, high energy electrodes for next-generation batteries, requires better understanding of how electrodes function by real time tracking of electrochemical reaction and the associated ionic transport in active constituents, preferentially at a range of relevant length scales due to the inherent heterogeneity of the electrodes. Most of the available in situ techniques, such as those based on hard X-ray scattering, are suited for bulk measurement at electrode level, but very often have no adequate spatial resolution to probe local structural changes in single particles or interfaces [1]. Highresolution scanning/transmission electron microscopy (S/TEM) - electron energy-loss spectroscopy (EELS) is powerful, not only for its high spatial resolution (down to sub- $\AA$ scale), but because of its analytical capability in imaging, diffraction, spectroscopy within a single instrument. The recent advances in designing in situ electrochemical cells for operation in the transmission electron microscope allow for direct visualization of the ionic transport and reaction within individual particles [1-4].

Figure 1 shows one type of electrochemical cell based on carbon-coated TEM grids, allowing for in situ $\mathrm{S} / \mathrm{TEM}$-EELS tracking of lithium transport in nanoparticles [3]. The carbon film, being used as the support of nanoparticles, also provides the pathway for electronic and ionic transport. The counter electrode, lithium metal coated with natively formed surface lithium oxynitride (acting as electrolyte), can be introduced into the TEM column using a piezo-driven biasing probe in the TEM sample stage. The electrode configuration of the cell was recently modified for probing local Li intercalation within a single particle of $\mathrm{Li}_{\mathrm{x}} \mathrm{FePO}_{4}$ (LFP) [5]. As illustrated in Figure 2a, through in situ electron diffraction and highresolution TEM imaging, details on the evolution of local Li concentration were revealed, showing that fast lithiation in nano-sized LFP proceeded via kinetically favorable solid solution transformation, accompanied with an unexpected reversal of Li concentration at the nanometer scale (Figure 2b). The findings from this study provide a new perspective on the local intercalation dynamics in battery electrodes.

Following an early in situ S/TEM-EELS study [3], the conversion reaction mechanisms were revisited through in situ crystallography of a single crystal of $\mathrm{FeF}_{2}$ upon lithiation (using the similar set up as in Figure 2a) [6]. Contradicting the traditional belief of causing structural breakdown, conversion in $\mathrm{FeF}_{2}$ was found to resemble an intercalation process - proceeding via topotactic reaction with the anion framework retained throughout the process. Similar phenomenon was observed in oxides and other types of conversion electrodes [7]. These findings provide a new paradigm for re-interpretation of conversion reaction and, for practical application, shed light on designing viable conversion electrodes for high energy batteries [8].

\section{References}

[1] H Wang, F Wang, "In-Situ, Operando Measurements of Rechargeable Batteries", Current Opinion in Chemical Engineering 13, 170 (2016)

[2] J.Y. Huang, et al., "In situ observation of the electrochemical lithiation of a single $\mathrm{SnO}_{2}$ nanowire electrode. Science 330, 1515 (2010). 
[3] F. Wang, et al., "Tracking of Li Transport and electrochemical reaction in nanoparticles", Nat. Commun. 3, 1201 (2012).

[4] C.M. Wang. "In situ transmission electron microscopy and spectroscopy studies of rechargeable batteries under dynamic operating conditions: A retrospective and perspective view." J. Mater. Res. 30, 326 (2015). [5] W. Zhang et al., "Localized concentration reversal of lithium during intercalation into nanoparticles", Science Advances 4, eaao2608 (2018).

[6] K. Karki, et al. "Revisiting Conversion Reaction Mechanisms in Lithium Batteries: Lithiation-driven Topotactic Transformation in $\mathrm{FeF}_{2}$ ", J. Am. Chem. Soc., 140, 17915 (2018).

[7] W. Zhang et al., "Multi-Electron Transfer Enabled by Topotactic Reaction in Magnetite", Nat. Commun. (accepted).

[8] This work is financially supported by the Laboratory Directed Research and Development (LDRD) program at Brookhaven National Laboratory and $\mathrm{m} 2 \mathrm{M} / \mathrm{t}$ EFRC Center.

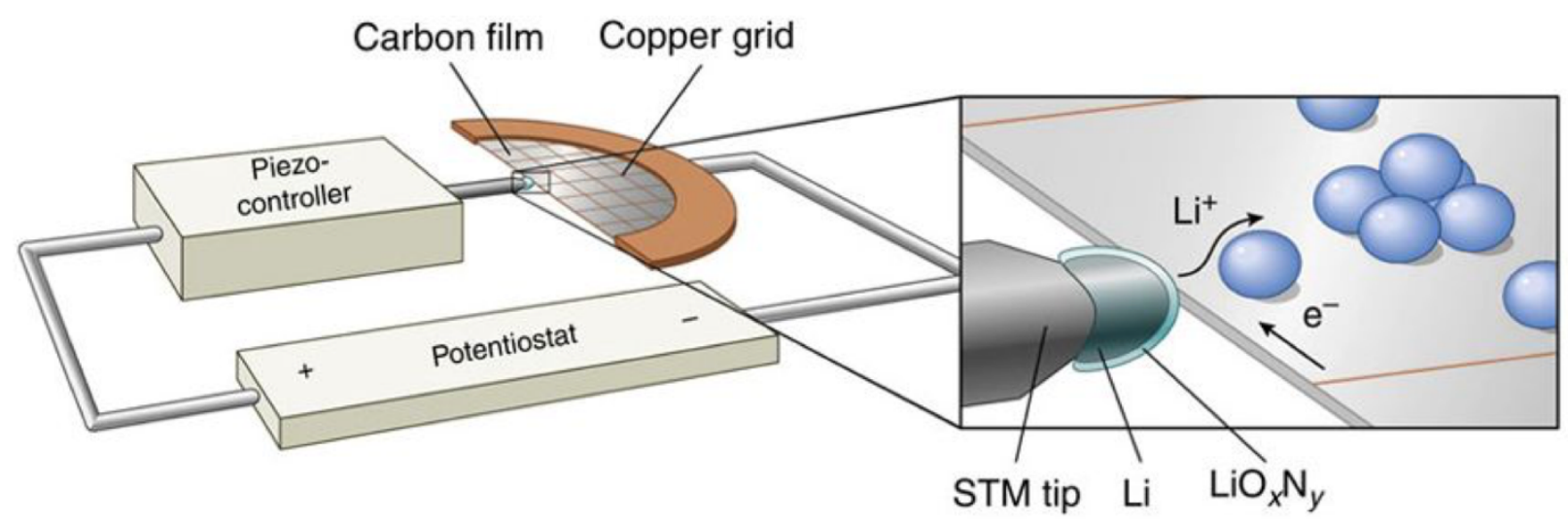

Figure 1. Schematic illustration of the electrochemical cell for in-situ S/TEM-EELS measurements [3].
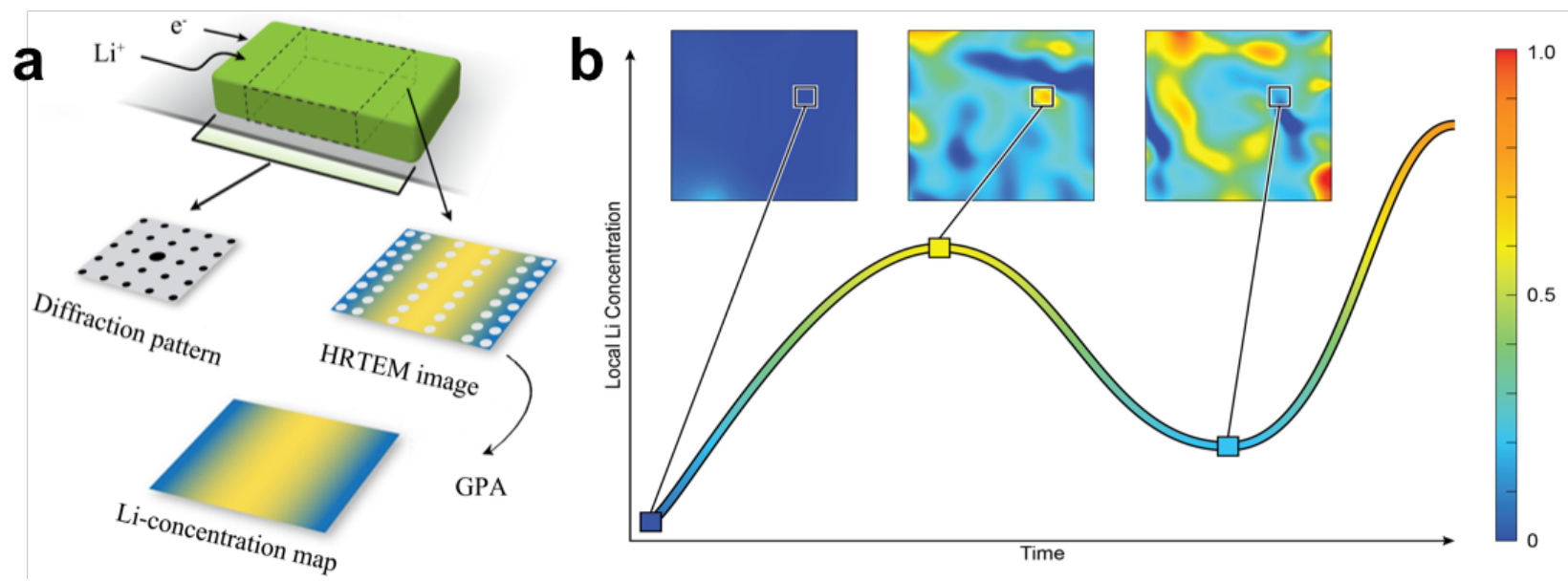

Figure 2. Real time tracking of lithium intercalation in a single $\mathrm{Li}_{x} \mathrm{FePO}_{4}$ (LFP) nanoparticle (a) Schematic illustration of the setup. (b) Maps of local lithium concentration within a single particle at intermediate lithiated states [5]. 